\title{
On the real cohomology of spaces of free loops on manifolds
}

\author{
by
}

\author{
Katsuhiko K u ri bay a s h i (Okayama)
}

\begin{abstract}
Let $L X$ be the space of free loops on a simply connected manifold $X$. When the real cohomology of $X$ is a tensor product of algebras generated by a single element, we determine the algebra structure of the real cohomology of $L X$ by using the cyclic bar complex of the de Rham complex $\Omega(X)$ of $X$. In consequence, the algebra generators of the real cohomology of $L X$ can be represented by differential forms on $L X$ through Chen's iterated integral map. Let $\mathbb{T}$ be the circle group. The $\mathbb{T}$-equivariant cohomology of $L X$ is also studied in terms of the cyclic homology of $\Omega(X)$.
\end{abstract}

Introduction. Let $X$ be a simply connected finite-dimensional manifold whose real cohomology is a tensor product of truncated polynomial algebras and exterior algebras. We call such a commutative algebra a TE-algebra. Let $L X$ be the space of free loops on $X$, that is, the space of all smooth maps from the circle group $\mathbb{T}$ to $X$. The purpose of this paper is to determine the algebra structures of the real cohomology of $L X$ when the real cohomology ring of $X$ is a TE-algebra, and of the $\mathbb{T}$-equivariant real cohomology of $L X$ when the real cohomology of $X$ is isomorphic to that of a sphere. Moreover, we will represent generators of the real cohomology and of the $\mathbb{T}$-equivariant real cohomology of $L X$ by explicit elements in the Hochschild homology and in the cyclic homology of the de Rham complex of $X$ respectively.

Let $X$ be a simply connected space and $\mathcal{F}(X)$ the fiber square

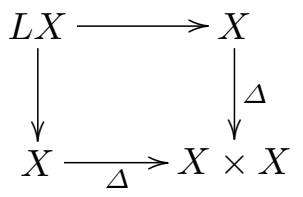

where $\Delta$ is the diagonal map. In the case where $X$ is not a manifold, we regard $L X$ as the space of all continuous loops on $X$. Let $\mathbf{k}$ be a field of

1991 Mathematics Subject Classification: 55N35, 55P62, 57N65, $19 \mathrm{D} 10$. 
characteristic zero. In [21], L. Smith has explicitly constructed a projective resolution, which is called a Koszul type resolution, of a graded complete intersection (GCI) algebra $\Lambda$ over $\mathbf{k}$ as a $\Lambda \otimes \Lambda$-module and used it to calculate $\operatorname{Tor}_{\Lambda \otimes \Lambda}(\Lambda, \Lambda)$. The Koszul type resolution and the Eilenberg-Moore spectral sequence of $\mathcal{F}(X)$ are relevant to the study of the space $L X$. For instance, by applying the result [22, Proposition 4.4.5] of M. Vigué-Poirrier to our case, we obtain

TheOrem A. Let $X$ be simply connected and formal. Then

$$
H^{*}(L X ; \mathbf{k}) \cong \operatorname{Tot} \operatorname{Tor}_{H^{*}}^{*, *}(X ; \mathbf{k}) \otimes H^{*}(X ; \mathbf{k})\left(H^{*}(X ; \mathbf{k}), H^{*}(X ; \mathbf{k})\right)
$$

as algebras.

For example, let $G$ be a compact connected Lie group and $H$ a maximal rank subgroup of $G$. Since the homogeneous space $G / H$ is formal, we can express the algebra $H^{*}(L(G / H) ; \mathbf{k})$ via the torsion functor.

Let $X$ be a simply connected manifold. Chen's iterated integral map $\sigma$ ([7]) may be regarded as a de Rham version of the Eilenberg-Moore map ([20], [19]) because $\sigma$ induces an algebra isomorphism from the Hochschild homology of the de Rham complex $\Omega(X)$ of $X$ to the de Rham cohomology $H_{\text {de Rham }}^{*}(L X)$. Since the Hochschild complex of $\Omega(X)$ is a double complex, in consequence, we obtain a spectral sequence converging to $H^{*}(L X ; \mathbb{R})$. We call it the Hochschild spectral sequence. When $H^{*}(X ; \mathbb{R})$ is a TE-algebra, by virtue of the Hochschild spectral sequence and Theorem $\mathrm{A}$, we get an explicit form of the algebra $H^{*}(L X ; \mathbb{R})$. Moreover, all generators of the cohomology can be represented by differential forms on $L X$ which are images by the iterated integral map (Theorem 2.1).

Cyclic homology groups defined by A. Connes for any associative algebra have been studied and generalized in [5], [10], [12] and [16]. In particular, T. G. Goodwillie [10] has extended Connes's construction to differential graded algebras (DGAs). J. D. S. Jones [12] has studied the cyclic homology theory for DGAs. One of his results asserts that the cyclic homology group for the singular complex $S^{*}(X)$ is isomorphic to the $\mathbb{T}$-equivariant cohomology of $L X$. A de Rham version of the result has been shown by E. Getzler, J. D. S. Jones and S. Petrack [9]: the cyclic homology of $\Omega(X)$ induced from the cyclic bar complex or its normalized complex is isomorphic to the homology of a de Rham model for $\mathbb{T}$-equivariant differential forms on $L X$ by the isomorphism induced from the iterated integral map, where the de Rham model is larger than the usual one (see [2], [17]), but equivalent. They have also given an $A_{\infty}$-algebra structure on the normalized cyclic bar complex of $\Omega(X)$ and on the de Rham model and shown that the iterated integral map is a morphism of $A_{\infty}$-algebras. Therefore we see that the spectral sequence (e.g. [10, II.2.4]) which is constructed from the normalized com- 
plex converges to the $\mathbb{T}$-equivariant real cohomology of $L X$ as an algebra. The spectral sequence enables us to consider the cohomology $H_{\mathbb{T}}^{*}(L X ; \mathbb{R})$ in terms of cyclic homology theory. In consequence, when $H^{*}(X ; \mathbb{R})$ is isomorphic to $H^{*}\left(S^{q} ; \mathbb{R}\right)$, we can determine the algebra structure of $H_{\mathbb{T}}^{*}(L X ; \mathbb{R})$ and represent all the algebra generators of $H_{\mathbb{T}}^{*}(L X ; \mathbb{R})$ by elements of the cyclic homology of $\Omega(X)$ through the iterated integral map.

The algebra structure of the cohomology and $\mathbb{T}$-equivariant cohomology of the space of free loops on a simply connected space whose rational cohomology is a GCI-algebra was studied in [24] and [1]. Since every TE-algebra is a GCI-algebra, our results about the algebra structure of $H^{*}(L X ; \mathbb{R})$ and $H_{\mathbb{T}}^{*}(L X ; \mathbb{R})$ are not new. The novelty here is that the generators of the algebras $H^{*}(L X ; \mathbb{R})$ and $H_{\mathbb{T}}^{*}(L X ; \mathbb{R})$ are represented by explicit elements in the cyclic bar complex of the de Rham complex of $X$ and so by differential forms on $L X$ through the iterated integral map.

The paper is organized as follows. In $\S 1$, we recall some results of [9]. Our results are stated in $\S 2$. In $\S 3$, we prepare a lemma to determine $\operatorname{Tor}_{\Lambda \otimes \Lambda}(\Lambda, \Lambda)$ as an algebra whenever $\Lambda$ is a TE-algebra. Moreover, an isomorphism from the Hochschild homology to $\operatorname{Tor}_{\Lambda \otimes \Lambda}(\Lambda, \Lambda)$ is given explicitly. $\S 4$ and $\S 5$ are devoted to proving our theorems completely.

The author wishes to thank Akira Kono for helpful conversations and for pointing out errors in the first version of the manuscript.

1. The iterated integral map. In order to explain the result of [9] more carefully, we recall the definitions of the (normalized) cyclic bar complex, the de Rham model of $\mathbb{T}$-equivariant differential forms on $L X$ and the iterated integral map.

The cyclic bar complex $\mathbf{C}(\Omega(X))$ of the de Rham complex $(\Omega(X), d)$ has three operators $b_{0}, b_{1}$ and $B$ which are called the exterior differential, the Hochschild boundary operator and the Connes coboundary operator respectively. The complex $\mathbf{C}(\Omega(X))$ is defined as follows:

$$
\begin{gathered}
\mathbf{C}(\Omega(X))=\sum_{k=0}^{\infty} \Omega(X) \otimes \Omega(X)^{\otimes k} \\
\operatorname{deg}\left(\omega_{0}, \ldots, \omega_{k}\right)=\operatorname{deg} \omega_{0}+\ldots+\operatorname{deg} \omega_{k}-k \quad \text { for }\left(\omega_{0}, \ldots, \omega_{k}\right) \in \mathbf{C}(\Omega(X)) \\
b_{0}\left(\omega_{0}, \ldots, \omega_{k}\right)=-\sum_{i=0}^{k}(-1)^{\varepsilon_{i-1}}\left(\omega_{0}, \ldots, \omega_{i-1}, d \omega_{i}, \omega_{i+1}, \ldots, \omega_{k}\right) \\
b_{1}\left(\omega_{0}, \ldots, \omega_{k}\right)=-\sum_{i=0}^{k-1}(-1)^{\varepsilon_{i}}\left(\omega_{0}, \ldots, \omega_{i-1}, \omega_{i} \omega_{i+1}, \omega_{i+2}, \ldots, \omega_{k}\right) \\
+(-1)^{\left(\operatorname{deg} \omega_{k}-1\right) \varepsilon_{k-1}}\left(\omega_{k} \omega_{0}, \ldots, \omega_{k-1}\right)
\end{gathered}
$$


and

$$
\begin{aligned}
B\left(\omega_{0}, \ldots, \omega_{k}\right)= & \sum_{i=0}^{k}(-1)^{\left(\varepsilon_{i-1}+1\right)\left(\varepsilon_{k}-\varepsilon_{i-1}\right)}\left(1, \omega_{i}, \ldots, \omega_{k}, \omega_{0}, \ldots, \omega_{i-1}\right) \\
& -\sum_{i=0}^{k}(-1)^{\left(\varepsilon_{i-1}+1\right)\left(\varepsilon_{k}-\varepsilon_{i-1}\right)}\left(\omega_{i}, \ldots, \omega_{k}, \omega_{0}, \ldots, \omega_{i-1}, 1\right),
\end{aligned}
$$

where $\varepsilon_{i}=\operatorname{deg} \omega_{0}+\ldots+\operatorname{deg} \omega_{i}-i$.

Let $b=b_{0}+b_{1}$ be the total boundary operator on $\mathbf{C}(\Omega(X))$. The operators $b$ and $B$ satisfy the formulas $b^{2}=b B+B b=B^{2}=0$.

Let $\mathbf{D}(\Omega(X))$ be the subspace of $\mathbf{C}(\Omega(X))$ generated by the image of the operators $S_{i}(f)$ and $b S_{i}(f)+S_{i}(f) b$, where $f \in \Omega^{0}(X)$ and $S_{i}(f)\left(\omega_{0}, \ldots, \omega_{k}\right)$ $=\left(\omega_{0}, \ldots, \omega_{i-1}, f, \omega_{i}, \ldots, \omega_{k}\right), i \geq 1$. The normalized cyclic bar complex $\mathbf{N}(\Omega(X))$ is the quotient complex $\mathbf{C}(\Omega(X)) / \mathbf{D}(\Omega(X))$.

To describe the main theorem of [9], we recall the definition of the iterated integral map. Let $\varphi_{t}(t \in \mathbb{T})$ be the circle action on $L X$, generated by the vector field $T$, and $\iota$ the interior product with $T$. Let $e_{t}: L X \rightarrow X$ denote the evaluation map at time $t$. The iterated integral map $\sigma: \mathbf{N}(\Omega(X)) \rightarrow$ $\Omega(L X)$ is defined by

$$
\sigma\left(\omega_{0}, \ldots, \omega_{k}\right)=\int_{\Delta_{k}} \omega_{0}(0) \wedge \iota \omega_{1}\left(t_{1}\right) \wedge \ldots \wedge \iota \omega_{k}\left(t_{k}\right) d t_{1} \ldots d t_{k},
$$

where $\Delta_{k}$ is the $k$-simplex $\left\{\left(t_{1}, \ldots, t_{k}\right) \in \mathbb{R}^{k} \mid 0 \leq t_{1} \leq \ldots \leq t_{k} \leq 1\right\}$ and $\omega(t)=e_{t}^{*} \omega$. Under the above notations and definitions, the main result of $[9]$ is stated as follows.

Theorem 1.1 [9, Theorem A, Theorem 3.1, Proposition 4.1]. Suppose that $X$ is a simply connected finite-dimensional manifold. Then

(1) the iterated integral map defines morphisms of DGAs

$$
(\mathbf{C}(\Omega(X)), b) \rightarrow(\mathbf{N}(\Omega(X)), b) \stackrel{\sigma}{\rightarrow}(\Omega(L X), d),
$$

and these induce isomorphisms on cohomology,

(2) the iterated integral map

$$
\sigma:(\mathbf{N}(\Omega(X))[u], b+u B) \rightarrow(\Omega(L X)[u], d+u \widetilde{P})
$$

is a morphism of $A^{\infty}$-algebras and an isomorphism on cohomology, where $\widetilde{P}(\omega)=\int_{0}^{1} \iota \varphi_{t}^{*} \omega d t$.

We merely need the algebra structure of $\mathbf{N}(\Omega(X))[u]$ and $\Omega(L X)[u]$. For details of the $A^{\infty}$-algebra structure, see [9] or [8].

Note that the algebra structures of $(\mathbf{C}(\Omega(X)), b)$ and $(\mathbf{N}(\Omega(X)), b)$ are given by the shuffle product $S$ : 
$S(\alpha, \beta)$

$=(-1)^{\operatorname{deg} \beta_{0}\left(\operatorname{deg} \alpha_{1}+\ldots+\operatorname{deg} \alpha_{p}-p\right)} \sum_{\sigma:(p, q) \text {-shuffle }}(-1)^{s(\sigma)}\left(\alpha_{0} \beta_{0}, \xi_{\sigma(1)}, \ldots, \xi_{\sigma(p+q)}\right)$,

where $\alpha=\left(\alpha_{0}, \ldots, \alpha_{p}\right), \beta=\left(\beta_{0}, \ldots, \beta_{q}\right),\left(\xi_{1}, \ldots, \xi_{p+q}\right)=\left(\alpha_{1}, \ldots, \alpha_{p}\right.$, $\left.\beta_{1}, \ldots, \beta_{q}\right)$ and $s(\sigma)=\sum\left(\operatorname{deg} \xi_{i}+1\right)\left(\operatorname{deg} \xi_{p+j}+1\right)$, summed over all pairs $(i, p+j)$ with $\sigma(i)>\sigma(p+j), 1 \leq i \leq p, 1 \leq j \leq q$.

Although the usual de Rham model of $\mathbb{T}$-equivariant differential forms on $L X$ is the complex $\left(\Omega(L X)^{\mathbb{T}}[u], d+u \iota\right)$, since the inclusion map $i$ : $\left(\Omega(L X)^{\mathbb{T}}[u], d+u \iota\right) \rightarrow(\Omega(L X)[u], d+u \widetilde{P})$ is a morphism of algebras and induces an isomorphism on cohomology, we use $(\Omega(L X)[u], d+u \widetilde{P})$ as a complex which defines the $\mathbb{T}$-equivariant cohomology $H_{\text {de Rham, } \mathbb{T}}^{*}(L X)$.

From the considerations of Beggs [3, Note 6.8], we obtain $H^{*}(L X) \cong$ $H_{\mathrm{de} \mathrm{Rham}}^{*}(L X)$ as algebras and $H_{\mathbb{T}}^{*}(L X) \cong H_{\mathrm{deRham}, \mathbb{T}}^{*}(L X)$ as algebras and as $H^{*}\left(B_{\mathbb{T}}\right)=\mathbb{R}[u]$-modules. In consequence, we have two isomorphisms of algebras:

$$
H(\mathbf{C}(\Omega(X)), b) \stackrel{H(\sigma)}{\longrightarrow} H^{*}(L X) \stackrel{H(\theta)}{\longleftarrow} \operatorname{Tor}_{S^{*}(X \times X)}^{*}\left(S^{*}(X), S^{*}(X)\right),
$$

where $\sigma$ and $\theta$ are the iterated integral map and the Eilenberg-Moore map ([20], [19]) respectively. So we obtain two methods to determine the algebra structure of $H^{*}(L X)$. One method is to calculate the cyclic bar complex. The other method is an application of the Eilenberg-Moore spectral sequence which has been used by L. Smith [21]. In explicit calculations of the Hochschild homology and cyclic cohomology, spectral sequences stated below are useful. Since $\left(\mathbf{C}(\Omega(X)), b_{0}, b_{1}\right)$ is regarded as a filtered double complex such that

$$
(\mathbf{C}(\Omega(X)))^{-p, q}=\left[\Omega(X) \otimes \Omega(X)^{\otimes p}\right]^{q}
$$

and

$$
F^{p}(\mathbf{C}(\Omega(X)))^{n}=\sum_{\substack{-i+j=n \\-i \geq p}}\left[\Omega(X) \otimes \Omega(X)^{\otimes i}\right]^{j},
$$

we can construct a spectral sequence ([4], [19]) converging to $H(\mathbf{C}(\Omega(X)), b)$ $\cong H_{\text {de Rham }}^{*}(L X)$. From the definition of the filtration of $\mathbf{C}(\Omega(X))$, we see that the $E_{2}$-term of the spectral sequence is isomorphic to the Hochschild homology $H\left(\mathbf{C}\left(H_{\text {de Rham }}^{*}(X)\right), b_{1}\right)$. The filtration of the complex $\mathbf{C}(\Omega(X))$ also respects the algebra structure. Hence we conclude that the spectral sequence converges to its target as an algebra and that the isomorphism from the $E_{2}$-term to the Hochschild homology is a morphism of algebras. Similarly from the filtered double complex $(\mathbf{N}(\Omega(X))[u], b, u B)$ such that

$$
(\mathbf{N}(\Omega(X))[u])^{p, q}=\mathbb{R}[u]^{2 p} \otimes \mathbf{N}^{q-p}(\Omega(X))
$$


and

$$
F^{p}(\mathbf{N}(\Omega(X))[u])^{n}=\sum_{\substack{i+j=n \\ i \geq p}} \mathbb{R}[u]^{2 i} \otimes \mathbf{N}^{j-i}(\Omega(X))
$$

we can obtain another spectral sequence [10, II.2.4] converging to $H(\mathbf{N}(\Omega(X))[u], b+u B)$, that is, to $H_{\text {de Rham, }}^{*}(L X)$ as an algebra. We may call the spectral sequences the Hochschild spectral sequence and the cyclic spectral sequence associated with $\Omega(X)$ respectively.

To be exact, the homology $H(\mathbf{N}(\Omega(X))[u], b+u B)$ is equal to the negative cyclic homology of the differential graded algebra (DGA) $A^{*}$ defined by $A^{-i}=\Omega^{i}(X): H^{*}(\mathbf{N}(\Omega(X))[u], b+u B)=H C_{-*}^{-}(A)$ (see [8]). However, we will call $H(\mathbf{N}(\Omega(X))[u], b+u B)$ the cyclic homology of $\Omega(X)$ in this paper.

2. Results. Let $\Gamma_{\mathbf{k}}$ be a TE-algebra, that is,

$$
\Gamma_{\mathbf{k}}=\Lambda\left(y_{1}, \ldots, y_{n}\right) \otimes \mathbf{k}\left[x_{1}, \ldots, x_{m}\right] /\left(x_{1}^{s_{1}+1}, \ldots, x_{m}^{s_{m}+1}\right) .
$$

Let $A$ be a ring and $A[\omega]$ an $A$-coefficient polynomial ring. We denote by $A[\omega] \geq 1$ the subalgebra of $A[\omega]$ consisting of polynomials whose constant term is zero. We will denote algebra generators of $H^{*}(X ; \mathbb{R})$ and their representatives with the same notations. By considering the Hochschild spectral sequence associated with the de Rham complex $\Omega(X)$, we have

THEOREM 2.1. Let $X$ be a simply connected manifold whose real cohomology is a TE-algebra $\Gamma_{\mathbb{R}}$. Let $\varrho_{i}$ be an element of the de Rham complex $\Omega(X)$ satisfying $d\left(\varrho_{i}\right)=x_{i}^{s_{i}+1}$. Then there exists an isomorphism of algebras

$$
\begin{aligned}
\varphi: H & :=\bigotimes_{j=1}^{n}\left\{\Lambda\left(y_{j}\right) \otimes \mathbb{R}\left[\left(1, y_{j}\right)\right]\right\} \otimes \bigotimes_{i=1}^{m}\left\{\mathbb{R}\left[x_{i}\right] /\left(x_{i}^{s_{i}+1}\right) \otimes \Lambda\left(\left(1, x_{i}\right)\right)\right. \\
& \left.\oplus\left(x_{i},\left(1, x_{i}\right)\right)\left[\alpha_{i}\right] \geq 1 /\left(\left(s_{i}+1\right) x_{i}^{s_{i}}\left(1, x_{i}\right)\right)\left[\alpha_{i}\right]\right\} \\
& \rightarrow H(\mathbf{C}(\Omega(X)), b) \cong H(\mathbf{N}(\Omega(X)), b)
\end{aligned}
$$

such that $\varphi(z)=z, \varphi((1, z))=(1, z)$,

$$
\begin{aligned}
\varphi\left(x_{i} \alpha_{i}^{k}\right) & =x_{i} \alpha_{i}^{k}-\sum_{p=1}^{k} k(k-1) \ldots(k-p+1) \gamma_{i, p} \alpha_{i}^{k-p}, \\
\varphi\left(\left(1, x_{i}\right) \alpha_{i}^{k}\right) & =\left(1, x_{i}\right) \alpha_{i}^{k}+\sum_{p=1}^{k} k(k-1) \ldots(k-p+1) \zeta_{i, p+1} \alpha_{i}^{k-p},
\end{aligned}
$$

where $z=x_{i}$ or $y_{j}$, and

$$
\alpha_{i}=\left(x_{i}^{s_{i}-1}, x_{i}, x_{i}\right)+\left(x_{i}^{s_{i}-2}, x_{i}^{2}, x_{i}\right)+\left(1, x_{i}^{s_{i}}, x_{i}\right) \in \mathbf{C}(\Omega(X)),
$$




$$
\begin{aligned}
\zeta_{i, p} & =\sum_{j=1}^{p}(1, \varrho_{i}, \ldots, \varrho_{i}, \overbrace{x_{i}}^{j t h}, \varrho_{i}, \ldots, \varrho_{i}) \in \mathbf{C}^{p, *}(\Omega(X)), \\
\gamma_{i, p} & =\left(s_{i}+1\right) \varrho_{i} \zeta_{i, p}-\left(x_{i}, \varrho_{i}, \ldots, \varrho_{i}\right) \in \mathbf{C}^{p, *}(\Omega(X)) .
\end{aligned}
$$

From Theorems 1.1 and 2.1, we have

Corollary 2.2. As algebras,

$$
H^{*}(L X ; \mathbb{R}) \cong H_{\text {de Rham }}^{*}(L X) \cong H(\mathbf{C}(\Omega(X)), b) \cong H .
$$

We can find some algebra generators of $H_{\mathbb{T}}^{*}(L X ; \mathbb{R}) \cong H_{\text {de Rham }, \mathbb{T}}^{*}(L X)$ by using the cyclic spectral sequence and Theorem 2.1.

Proposition 2.3. Let $X$ be a manifold satisfying the condition in Theorem 2.1. Then there exists a monomorphism of algebras and $\mathbb{R}[u]$-modules $i: \mathbb{R}[u] \otimes \mathbb{R}\left[v_{1}, \ldots, v_{n}\right] \otimes \Lambda\left(\nu_{1}, \ldots, \nu_{m}\right) /\left(v_{j} u, \nu_{i} u ; 1 \leq j \leq n, 1 \leq i \leq m\right)$

$$
\rightarrow H_{\text {de Rham }, \mathbb{T}}^{*}(L X) \cong H_{\mathbb{T}}^{*}(L X ; \mathbb{R})
$$

such that $i\left(v_{j}\right)=\int_{0}^{1} \iota \omega_{t}^{*} y_{j} d t$ and $i\left(\nu_{i}\right)=\int_{0}^{1} \iota \omega_{t}^{*} x_{i} d t$.

In Proposition 2.3 , when $m=0$, that is, $H^{*}(X ; \mathbb{R})$ is an exterior algebra, we see that the morphism $i$ is an isomorphism if and only if $n=1$. The result is obtained by calculating $H_{\mathbb{T}}^{*}\left(L S^{2 l-1} ; \mathbb{R}\right)$. By using the cyclic spectral sequence and Theorem 2.1, we can determine the algebra structure of $H_{\mathbb{T}}^{*}\left(L S^{2 l} ; \mathbb{R}\right)$.

THEOREM 2.4. Let $X$ be a simply connected manifold whose real cohomology is isomorphic to that of a sphere $S^{q}$.

(1) If $q=2 l-1$, there exists an isomorphism of algebras and $\mathbb{R}[u]$ modules

$$
\mathbb{R}[u] \otimes \mathbb{R}[(1, y)] /((1, y) u) \stackrel{\varphi_{2 l-1}}{\longrightarrow} H(\mathbf{N}(\Omega(X))[u], b+u B)
$$

such that $\varphi_{2 l-1}((1, y))=(1, y)$ and $\varphi_{2 l-1}(u)=u$, where $\operatorname{deg} y=2 l-1$.

(2) If $q=2 l$, there exists an isomorphism of algebras and $\mathbb{R}[u]$-modules

$$
\begin{aligned}
\mathbb{R}[u] \otimes\left\{\Lambda((1, x)) \oplus((1, x))[\alpha]^{\geq 1}\right\} /\left((1, x) \alpha^{k} u ; k\right. & \geq 0) \\
& \stackrel{\varphi_{2 l}}{\longrightarrow} H(\mathbf{N}(\Omega(X))[u], b+u B)
\end{aligned}
$$

such that $\varphi_{2 l}((1, x))=(1, x), \varphi_{2 l}(u)=u$ and

$$
\varphi_{2 l}\left((1, x) \alpha^{k}\right)=(1, x) \alpha^{k}+\sum_{p=1}^{k} k(k-1) \ldots(k-p+1) \zeta_{p+1} \alpha^{k-p},
$$

where $\operatorname{deg} x=2 l$ and $\alpha=(1, x, x)$.

If the reduced cyclic homology of $\Omega(X)$ is non-zero, then the cyclic homology of the algebra is not a free $\mathbb{R}[u]$-module [23, Théorème 1]. So it is 
not easy to determine an explicit algebra structure of the cyclic homology $H(\mathbf{N}(\Omega(X))[u], b+u B)$ from the Künneth theorem [11, Theorem 3.1] and Theorem 2.4 even if $H^{*}(X ; \mathbb{R})$ is isomorphic to the algebra $\Gamma_{\mathbb{R}}$. In a further article [15], it is clarified that the algebra structure of $H(\mathbf{N}(\Omega(X))[u], b+u B)$ can be represented by the Hochschild homology of $\Omega(X)$ and the LodayQuillen *-product.

3. Homological algebra. Let $\Lambda$ be a non-negatively graded connected commutative algebra over a field $\mathbf{k}$ of characteristic zero. Let $\mathcal{K}$ denote a DGA $\Lambda \otimes \mathbf{k}[\omega]$ equipped with a differential $d$ satisfying $d(\omega) \in \Lambda$ and $d(\lambda)=0$ for any $\lambda \in \Lambda$.

LEMma 3.1. We have

$$
H(\mathcal{K}, d) \cong \Lambda \oplus \operatorname{Ann}(d \omega)[\omega]^{\geq 1} /(d \omega)[\omega]
$$

as algebras, where $\operatorname{Ann}(d \omega)$ is the ideal of $\Lambda$ which annihilates $d \omega$, and $(d \omega)$ is the ideal of $\Lambda$ generated by $d \omega$.

Proof. For any $\sum_{i=0}^{n} a_{i} \omega^{i} \in \operatorname{Ker} d$,

$$
0=d\left(\sum_{i=0}^{n} a_{i} \omega^{i}\right)=\sum_{i=1}^{n}(-1)^{\operatorname{deg} a_{i}} i a_{i} d \omega \omega^{i-1} .
$$

Therefore $\sum_{i=0}^{n} a_{i} \omega^{i} \in \Lambda \oplus \operatorname{Ann}(d \omega)[\omega]^{\geq 1}$. For any $a=\sum_{i=0}^{n} a_{i} d \omega \omega^{i} \in$ $(d \omega)[\omega]$, there exists $\beta$ such that $d(\beta)=a$. In fact, we can take

$$
\beta=(-1)^{\operatorname{deg} a_{0}} a_{0} \omega+\sum_{i=1}^{n} \frac{(-1)^{\operatorname{deg} a_{i}}}{i+1} a_{i} \omega^{i+1} .
$$

Clearly $(d \omega)[\omega]$ contains $\operatorname{Im} d$. Thus we have Lemma 3.1.

By applying Lemma 3.1 to the Koszul type resolution constructed by L. Smith [21], we have

Proposition 3.2. As bigraded algebras,

$$
\begin{array}{r}
\operatorname{Tor}_{\Gamma_{\mathbb{R}} \otimes \Gamma_{\mathbb{R}}}^{*, *}\left(\Gamma_{\mathbb{R}}, \Gamma_{\mathbb{R}}\right) \cong \bigotimes_{j=1}^{n}\left\{\Lambda\left(y_{j}\right) \otimes \mathbf{k}\left[\nu_{j}\right]\right\} \otimes \bigotimes_{i=1}^{m}\left\{\mathbf{k}\left[x_{i}\right] /\left(x_{i}^{s_{i}+1}\right) \otimes \Lambda\left(u_{i}\right)\right. \\
\left.\oplus\left(x_{i}, u_{i}\right)\left[\omega_{i}\right]^{\geq 1} /\left(\left(s_{i}+1\right) x_{i}^{s_{i}} u_{i}\right)\left[\omega_{i}\right]\right\} .
\end{array}
$$

We can consider the algebra structure on $\left(\mathbf{C}(\Lambda), b_{1}\right)$ induced from the shuffle product $S$ as that defined from a product on a projective resolution of $\Lambda$ as a $\Lambda \otimes \Lambda$-module. In order to describe this more precisely, we recall the standard resolution $(\mathcal{S}(\Lambda), \partial)$ of $\Lambda$ as a $\Lambda \otimes \Lambda$-module ([6], [18]). The 
resolution $(\mathcal{S}(\Lambda), \partial)$ is defined as follows:

$$
\mathcal{S}(\Lambda)=\Lambda \otimes \Lambda \otimes \widetilde{S}(\Lambda), \quad \widetilde{S}(\Lambda)=\sum_{k=1}^{\infty} \bar{\Lambda}^{\otimes k}
$$

and

$$
\begin{aligned}
\partial\left(\lambda_{0}, \xi, \lambda_{1}, \ldots,\right. & \left.\lambda_{n}\right) \\
= & -(-1)^{\operatorname{deg} \lambda_{1} \operatorname{deg} \xi+\operatorname{deg} \lambda_{0}+\operatorname{deg} \xi}\left(\lambda_{0} \lambda_{1}, \xi, \lambda_{2}, \ldots, \lambda_{n}\right) \\
& -\sum_{i=1}^{n-1}(-1)^{\varepsilon_{i}+\operatorname{deg} \xi}\left(\lambda_{0}, \xi, \lambda_{1}, \ldots, \lambda_{i} \lambda_{i+1}, \ldots, \lambda_{n}\right) \\
& +(-1)^{\left(\varepsilon_{n-1}+\operatorname{deg} \xi\right)\left(\operatorname{deg} \lambda_{n}+1\right)-\operatorname{deg} \lambda_{0} \operatorname{deg} \lambda_{n}}\left(\lambda_{0}, \lambda_{n} \xi, \lambda_{1}, \ldots, \lambda_{n-1}\right),
\end{aligned}
$$

where $\bar{\Lambda}=\{\lambda \in \Lambda \mid \operatorname{deg} \lambda>1\}$ and $\varepsilon_{i}=\operatorname{deg} \lambda_{0}+\ldots+\operatorname{deg} \lambda_{i}-i$.

Note that $\widetilde{S}(\Lambda)$ is regarded as a differential graded algebra [18] with the shuffle product

$$
\left(\alpha_{1}, \ldots, \alpha_{n}\right) *\left(\beta_{1}, \ldots, \beta_{m}\right)=\sum_{\sigma:(n, m) \text {-shuffle }}(-1)^{s(\sigma)}\left(\xi_{\sigma(1)}, \ldots, \xi_{\sigma(n+m)}\right),
$$

where $\left(\xi_{1}, \ldots, \xi_{p+q}\right)=\left(\alpha_{1}, \ldots, \alpha_{p}, \beta_{1}, \ldots, \beta_{q}\right)$. This enables us to conclude that $\left(\mathcal{S}(\Lambda), \partial, *^{\prime}\right)$ is a differential graded algebra with the product $*^{\prime}$ defined by

$$
\begin{aligned}
& \left(\alpha_{0}, \lambda, \alpha_{1}, \ldots, \alpha_{n}\right) *^{\prime}\left(\beta_{0}, \lambda^{\prime}, \beta_{1}, \ldots, \beta_{m}\right) \\
& \quad=(-1)^{\operatorname{deg} \beta_{0} \tilde{\varepsilon}_{n}+\operatorname{deg} \lambda \operatorname{deg} \beta_{0}+\operatorname{deg} \lambda^{\prime} \tilde{\varepsilon}_{n}}\left(\alpha_{0} \beta_{0}, \lambda \lambda^{\prime},\left(\alpha_{1}, \ldots, \alpha_{n}\right) *\left(\beta_{1}, \ldots, \beta_{m}\right)\right),
\end{aligned}
$$

where $\widetilde{\varepsilon}_{n}=\operatorname{deg} \alpha_{1}+\ldots+\operatorname{deg} \alpha_{n}-n$. Let $m: \Lambda \otimes \Lambda \rightarrow \Lambda$ be the product of $\Lambda$. Since the DGAs $\left(\Lambda \otimes{ }_{\Lambda \otimes \Lambda} \mathcal{S}(\Lambda), \pm 1 \otimes \partial, m \otimes *^{\prime}\right)$ and $\left(\Lambda \otimes \widetilde{S}(\Lambda), b_{1}, S\right)$ are isomorphic, it follows that $H\left(\mathbf{C}(\Lambda), b_{1}\right) \cong H\left(\Lambda \otimes \widetilde{S}(\Lambda), b_{1}\right) \cong \operatorname{Tor}_{\Lambda \otimes \Lambda}(\Lambda, \Lambda)$ as algebras. In particular, by choosing the cohomology ring $H^{*}(X ; \mathbb{R})$ of a simply connected manifold $X$ for $\Lambda$, we see that the $E_{2}$-term of the Hochschild spectral sequence associated with $\Omega(X)$ is isomorphic to

$$
\operatorname{Tor}_{H^{*}(X ; \mathbb{R}) \otimes H^{*}(X ; \mathbb{R})}^{*, *}\left(H^{*}(X ; \mathbb{R}), H^{*}(X ; \mathbb{R})\right)
$$

as an algebra. In order to represent elements in the spectral sequence by elements of $\mathbf{C}(\Omega(X))$, we need an explicit isomorphism from the Hochschild homology to $\operatorname{Tor}_{H^{*}(X ; \mathbb{R}) \otimes H^{*}(X ; \mathbb{R})}\left(H^{*}(X ; \mathbb{R}), H^{*}(X ; \mathbb{R})\right)$. It will be constructed in Proposition 3.4.

Re mark 3.3. Let $(\mathcal{S}(\Lambda), \partial)$ be the standard resolution of $\Lambda$ in the above argument and $(\mathcal{F}, d)$ another projective resolution of $\Lambda$ as a $\Lambda \otimes \Lambda$-module with a product $m_{\mathcal{F}}$. By the usual argument in homological algebra, we have a morphism $\Psi:(\mathcal{S}(\Lambda), \partial) \rightarrow(\mathcal{F}, d)$ of resolutions over the identity map id on $\Lambda$ which induces an isomorphism $\operatorname{Tor}_{\mathrm{id}} \otimes \mathrm{id}(\mathrm{id}, \Psi)$ from $H\left(\Lambda \otimes_{\Lambda \otimes \Lambda} \mathcal{S}(\Lambda), \pm 1 \otimes \partial\right)$ 
to $H(\Lambda \otimes \Lambda \otimes \Lambda \mathcal{F}, \pm 1 \otimes d)$ as $\Lambda$-modules. The $\Lambda \otimes \Lambda$-module $\Lambda$ is regarded as a $\Lambda \otimes \Lambda \otimes \Lambda \otimes \Lambda$-module with the multiplication $\widetilde{m}: \Lambda \otimes \Lambda \otimes \Lambda \otimes \Lambda \rightarrow \Lambda \otimes \Lambda$ defined by

$$
\widetilde{m}(a \otimes b \otimes c \otimes d)=(-1)^{\operatorname{deg} b \operatorname{deg} c} m(a \otimes c) \otimes m(b \otimes d) .
$$

Therefore, we can consider the resolutions $(\mathcal{S}(\Lambda), \partial)$ and $(\mathcal{F}, d)$ as resolutions of $\Lambda$ as a $\Lambda \otimes \Lambda \otimes \Lambda \otimes \Lambda$-module. Since $m_{\mathcal{F}} \circ(\Psi \otimes \Psi)$ and $\Psi \circ *^{\prime}$ are morphisms of projective resolutions of $\Lambda$ as a $\Lambda \otimes \Lambda \otimes \Lambda \otimes \Lambda$-module over $m: \Lambda \otimes \Lambda \rightarrow \Lambda$, it follows that

$$
\begin{aligned}
\operatorname{Tor}_{\tilde{m}}\left(m, m_{\mathcal{F}}\right) \operatorname{Tor}_{\mathrm{id}} \otimes \mathrm{id} \otimes \mathrm{id} \otimes \mathrm{id} & (\mathrm{id} \otimes \mathrm{id}, \Psi \otimes \Psi) \\
& =\operatorname{Tor}_{\tilde{m}}\left(m, m_{\mathcal{F}} \circ(\Psi \otimes \Psi)\right) \\
& =\operatorname{Tor}_{\tilde{m}}\left(m, \Psi \circ *^{\prime}\right)=\operatorname{Tor}_{\mathrm{id} \otimes \mathrm{id}}(\mathrm{id}, \Psi) \operatorname{Tor}_{\tilde{m}}\left(m, *^{\prime}\right) .
\end{aligned}
$$

It turns out that $\operatorname{Tor}_{\mathrm{id} \otimes \mathrm{id}}(\mathrm{id}, \Psi)$ is an isomorphism of algebras.

Let $(\mathcal{F}, d)$ be the Koszul type resolution ([21, Lemma 3.2], [14, Proposition 1.1]) whose differential $d$ is minus the original one.

Proposition 3.4. There exists a morphism of $\Gamma_{\mathbb{R}}$-modules

$$
\theta:\left(\Gamma_{\mathbb{R}} \otimes_{\Gamma_{\mathbb{R}} \otimes \Gamma_{\mathbb{R}}} \Gamma_{\mathbb{R}} \otimes \Gamma_{\mathbb{R}} \otimes \widetilde{S}\left(\Gamma_{\mathbb{R}}\right), \pm 1 \otimes \partial\right) \rightarrow\left(\Gamma_{\mathbb{R}} \otimes_{\Gamma_{\mathbb{R}} \otimes \Gamma_{\mathbb{R}}} \mathcal{F}, \pm 1 \otimes d\right)
$$

such that $\theta(\gamma)=\gamma$ for any $\gamma \in \Gamma_{\mathbb{R}}, \theta\left(\left(1, y_{j}\right)\right)=\nu_{j}, \theta\left(\left(1, x_{i}\right)\right)=u_{i}, \theta\left(\alpha_{i}\right)$ $=\omega_{i}$ and the induced map $H(\theta): H\left(\mathbf{C}\left(\Gamma_{\mathbb{R}}\right), b_{1}\right) \rightarrow \operatorname{Tor}_{\Gamma_{\mathbb{R}} \otimes \Gamma_{\mathbb{R}}}^{*, *}\left(\Gamma_{\mathbb{R}}, \Gamma_{\mathbb{R}}\right)$ is an isomorphism of algebras, where

$$
\alpha_{i}=\left(x_{i}^{s_{i}-1}, x_{i}, x_{i}\right)+\left(x_{i}^{s_{i}-2}, x_{i}^{2}, x_{i}\right)+\ldots+\left(1, x_{i}^{s_{i}}, x_{i}\right)
$$

and $\left(1, y_{j}\right),\left(1, x_{i}\right) \in \Gamma_{\mathbb{R}} \otimes \widetilde{S}\left(\Gamma_{\mathbb{R}}\right)=\Gamma_{\mathbb{R}} \otimes_{\Gamma_{\mathbb{R}} \otimes \Gamma_{\mathbb{R}}} \Gamma_{\mathbb{R}} \otimes \Gamma_{\mathbb{R}} \otimes \widetilde{S}\left(\Gamma_{\mathbb{R}}\right)$.

Pro of. To prove this proposition, we construct a morphism $\Psi=\left\{\psi_{-n}\right\}$ of resolutions of $\Gamma_{\mathbb{R}}$ as a $\Gamma_{\mathbb{R}} \otimes \Gamma_{\mathbb{R}}$-module

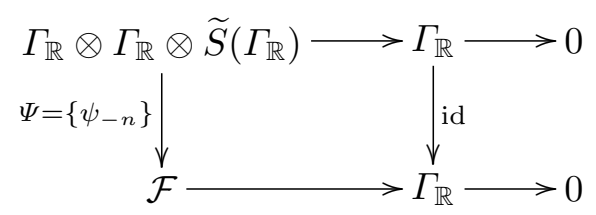

explicitly in low degrees of the resolutions. First, we define $\psi_{0}: \Gamma_{\mathbb{R}} \otimes \Gamma_{\mathbb{R}} \rightarrow$ $\Gamma_{\mathbb{R}} \otimes \Gamma_{\mathbb{R}}=\mathcal{F}^{0}$ to be the identity map. By demanding that $\left.d \psi_{-1}\right|_{\tilde{S}^{-1}\left(\Gamma_{\mathbb{R}}\right)}=$ $\psi_{0} \partial$, we define $\left.\psi_{-1}\right|_{\tilde{S}^{-1}\left(\Gamma_{\mathbb{R}}\right)}$ by $\psi_{-1}\left(\left(1,1, x_{i}^{k}\right)\right)=\zeta_{i, k} u_{i}$ and $\psi_{-1}\left(\left(1,1, y_{j}\right)\right)=$ $\nu_{j}$, where

$$
\zeta_{i, k}=\left(x_{i}^{k-1}, 1\right)+\left(x_{i}^{k-2}, x_{i}\right)+\ldots+\left(1, x_{i}^{k-1}\right)
$$

and $\zeta_{i, 1}=1$. Moreover, we can define $\psi_{-1}$ on $\Gamma_{\mathbb{R}} \otimes \Gamma_{\mathbb{R}} \otimes \widetilde{S}^{-1}\left(\Gamma_{\mathbb{R}}\right)$ as a morphism of $\Gamma_{\mathbb{R}} \otimes \Gamma_{\mathbb{R}}$-modules. Since $d, \partial$ and $\psi_{0}$ are morphisms of $\Gamma_{\mathbb{R}} \otimes \Gamma_{\mathbb{R}^{-}}$ 
modules, it follows that $d \psi_{-1}=\psi_{0} \partial$. Put

$$
\alpha_{i}=\left(1, x_{i}^{s_{i}-1}, x_{i}, x_{i}\right)+\left(1, x_{i}^{s_{i}-2}, x_{i}^{2}, x_{i}\right)+\ldots+\left(1,1, x_{i}^{s_{i}}, x_{i}\right) .
$$

We can verify that $\psi_{-1} \partial\left(\alpha_{i}\right)=d\left(\omega_{i}\right)$. Therefore, defining the map $\psi_{-2}$ : $\Gamma_{\mathbb{R}} \otimes \Gamma_{\mathbb{R}} \otimes \widetilde{S}^{-2}\left(\Gamma_{\mathbb{R}}\right) \rightarrow \mathcal{F}$ by $\psi_{-2}\left(\alpha_{i}\right)=\omega_{i}$, we see that $d \psi_{-2}=\psi_{-1} \partial$. Moreover, a morphism of resolutions $\Psi$ is obtained by extending the maps $\psi_{-n}(n=0,1$ and 2$)$. From the argument in Remark 3.3, we conclude that $\theta=\mathrm{id} \otimes \Psi$ is the required morphism of $\Gamma_{\mathbb{R}}$-modules.

4. Proof of Theorem 2.1. Let $X$ be a simply connected manifold whose cohomology is a GCI-algebra:

$$
H^{*}(X ; \mathbb{R}) \cong H_{\text {de Rham }}^{*}(X) \cong \Lambda\left(y_{1}, \ldots, y_{n}\right) \otimes \mathbf{k}\left[x_{1}, \ldots, x_{m}\right] /\left(\tau_{1}, \ldots, \tau_{m}\right) .
$$

We define a complex $\widetilde{\Omega}(X)$ as follows:

$$
\widetilde{\Omega}(X)=\Lambda\left(y_{1}, \ldots, y_{n}\right) \otimes \mathbf{k}\left[x_{1}, \ldots, x_{m}\right] \otimes \Lambda\left(\varrho_{1}, \ldots, \varrho_{m}\right),
$$

with $d\left(\varrho_{i}\right)=\tau_{i}$ and $d\left(y_{j}\right)=d\left(x_{i}\right)=0$. Let $\Phi$ be a well-defined homomorphism from $\widetilde{\Omega}(X)$ to $\Omega(X)$ defined by $\Phi\left(y_{j}\right)=y_{j}, \Phi\left(x_{i}\right)=x_{i}$ and $\Phi\left(\varrho_{i}\right)=\varrho_{i}$, where $x_{i}$ and $y_{j}$ in $\Omega(X)$ are representatives of $x_{i}$ and $y_{j}$ in $H^{*}(X ; \mathbb{R})$ respectively, and $\varrho_{i}$ in $\Omega(X)$ satisfies $d\left(\varrho_{i}\right)=\tau_{i}$. Since $\tau_{1}, \ldots, \tau_{m}$ is a regular sequence, it follows that $\Phi$ induces an isomorphism on cohomology. Thus we have a minimal model $(\widetilde{\Omega}(X), d)$ of $(\Omega(X), d)$. Moreover, we define a $\operatorname{map} h: \widetilde{\Omega}(X) \rightarrow H^{*}(X ; \mathbb{R})$ by $h\left(y_{j}\right)=y_{j}, h\left(x_{i}\right)=x_{i}$ and $h\left(\varrho_{i}\right)=0$. It is obvious that $h$ is a morphism of differential graded algebras and induces an isomorphism on cohomology. Therefore we see that a simply connected manifold whose cohomology is a GCI-algebra is formal. Since $H(\widetilde{\Omega}(X), b)$ and $H(\Omega(X), b)$ are isomorphic, we will consider the Hochschild spectral sequence of $\widetilde{\Omega}(X)$ instead of that of $\Omega(X)$.

The following proposition presents explicit closed elements representing algebra generators in the $E_{\infty}$-term of the Hochschild spectral sequence. We will also use the proposition to solve the extension problem of the spectral sequence.

Proposition 4.1. The elements

$$
\xi_{i, k}=x_{i} \alpha_{i}^{k}-\sum_{p=1}^{k} k(k-1) \ldots(k-p+1) \gamma_{i, p} \alpha_{i}^{k-p}
$$

and

$$
\eta_{i, k}=\left(1, x_{i}\right) \alpha_{i}^{k}+\sum_{p=1}^{k} k(k-1) \ldots(k-p+1) \zeta_{i, p+1} \alpha_{i}^{k-p} .
$$

are closed in $\mathbf{C}(\widetilde{\Omega}(X), b)$. 
Proposition 4.1 follows from

LEMMA 4.2. (1) $b_{1} \zeta_{i, p}=b_{1} \gamma_{i, p}=0$.

(2) $\left(1, \varrho_{i}\right)^{p-1} \zeta_{i, 1}=(p-1) ! \zeta_{i, p}$.

(3) $\zeta_{i, p} b_{1} \alpha_{i}=b_{0} \zeta_{i, p+1}$.

(4) $-\gamma_{i, p} b_{1} \alpha_{i}=b_{0} \gamma_{i, p+1}$.

P r o o f. It is straightforward to check (1). Since $\left(1, \varrho_{i}\right) \zeta_{i, p-1}=(p-1) \zeta_{i, p}$, we have (2). By using (2) and (3), we can verify (3) and (4) respectively.

Proof of Theorem 2.1. Let $\left\{E_{r}, d_{r}\right\}$ be the Hochschild spectral sequence of the DGA $\widetilde{\Omega}(X)$. By Proposition 3.4, we have

$$
\begin{aligned}
& E_{2}^{*, *} \cong H\left(\mathbf{C}\left(H^{*}(X)\right), b_{1}\right) \cong \operatorname{Tor}_{\Gamma_{\mathbb{R}}^{*} \otimes \Gamma_{\mathbb{R}}}^{* * *}\left(\Gamma_{\mathbb{R}}, \Gamma_{\mathbb{R}}\right) \\
& \cong \bigotimes_{j=1}^{n}\left\{\Lambda\left(y_{j}\right) \otimes \mathbf{k}\left[\left(1, y_{j}\right)\right]\right\} \otimes \bigotimes_{i=1}^{m}\left\{\mathbf{k}\left[x_{i}\right] /\left(x_{i}^{s_{i}+1}\right) \otimes \Lambda\left(\left(1, x_{i}\right)\right)\right. \\
&\left.\quad \oplus\left(x_{i},\left(1, x_{i}\right)\right)\left[\alpha_{i}\right]^{\geq 1} /\left(\left(s_{i}+1\right) x_{i}^{s_{i}}\left(1, x_{i}\right)\right)\left[\alpha_{i}\right]\right\} .
\end{aligned}
$$

Since the spectral sequence $\left\{E_{r}, d_{r}\right\}$ converges to the algebra $H^{*}(L X ; \mathbb{R}) \cong$ $H_{\text {de Rham }}^{*}(L X)$, from Theorem $\mathrm{A}$ it follows that $\left\{E_{r}, d_{r}\right\}$ collapses at the $E_{2}$-term: $E_{2} \cong E_{\infty} \cong E_{0}$. The elements $x_{i}, y_{j},\left(1, x_{i}\right)$ and $\left(1, y_{j}\right)$ are closed in $\mathbf{C}(\widetilde{\Omega}(X))$. Therefore we can take the elements $x_{i}, y_{j},\left(1, x_{i}\right)$ and $\left(1, y_{j}\right)$ from $H(\mathbf{C}(\widetilde{\Omega}(X)), b)$ as representatives of $x_{i}, y_{j},\left(1, x_{i}\right)$ and $\left(1, y_{j}\right)$ in $E_{0}^{*, *}$ respectively. Moreover, from Proposition 4.1, we can choose the closed elements $\xi_{i, k}$ and $\eta_{i, k}$ of $\mathbf{C}(\widetilde{\Omega}(X))$ as representatives of $x_{i} \alpha_{i}^{k}$ and $\left(1, x_{i}\right) \alpha_{i}^{k}$ in $E_{0}^{*, *}$ respectively. It remains to solve extension problems. We need to verify that

$$
\begin{aligned}
& x^{s_{i}} \cdot \xi_{i, k}=0, \\
& x^{s_{i}} \cdot \eta_{i, k}=0
\end{aligned}
$$

and

$$
\left(1, x_{i}\right) \cdot \eta_{i, k}=0
$$

in $H^{*}(L X ; \mathbb{R})$. Let $\Lambda_{i}$ be a DGA $\mathbb{R}\left[x_{i}\right] \otimes \Lambda\left(\varrho_{i}\right)$ equipped with a differential $d$ satisfying $d\left(\varrho_{i}\right)=x_{i}^{s_{i}+1}$. Since we can define a morphism $f: \Lambda_{i} \rightarrow \widetilde{\Omega}(X)$ of DGAs so that $f^{*}\left(x_{i}\right)=x_{i}, f^{*}\left(\left(1, x_{i}\right)\right)=\left(1, x_{i}\right), f^{*}\left(\xi_{i, k}\right)=\xi_{i}$ and $f^{*}\left(\eta_{i, k}\right)=\eta_{i, k}$ on Hochschild homology, it suffices to verify (4.1)-(4.3) in $H\left(\mathbf{C}\left(\Lambda_{i}\right), b\right)$ for any $i$, for solving the extension problems of $H(\mathbf{C}(\widetilde{\Omega}(X)), b)$. Since totdeg $x_{i}^{s_{i}} \cdot x_{i} \alpha_{i}^{k}-\operatorname{totdeg} x_{i}^{l} \alpha_{i}^{s}>0$ when $s_{i}+1>l$ and $k>s$ it follows that $x_{i}^{s_{i}} \cdot x_{i} \alpha_{i}^{k}=0$ in $H\left(\mathbf{C}\left(\Lambda_{i}\right), b\right)$. Similarly, we can verify that $x_{i}^{s_{i}} \cdot\left(1, x_{i}\right) \alpha_{i}^{k}=0$ in $H\left(\mathbf{C}\left(\Lambda_{i}\right), b\right)$. From Lemma 4.2(2), we have (4.3). This completes the proof. 


\section{Proof of Proposition 2.3 and Theorem 2.4}

Proof of Proposition 2.3. Let $\left\{E_{r}, d_{r}\right\}$ be the cyclic spectral sequence (see $\S 1$ ) associated with the DGA $\Omega(X)$. The spectral sequence converges to $H(\mathbf{N}(\Omega(X))[u], b+u B)$ as an algebra and satisfies

$$
E_{1}^{*, *} \cong \mathbb{R}[u] \otimes H(\mathbf{N}(\Omega(X)), b) \quad\left(E_{1}^{p, q} \cong \mathbb{R}[u]^{2 p} \otimes H^{q-p}(\mathbf{N}(\Omega(X)), b)\right)
$$

and $d_{1}=u B$, where $B$ is the Connes coboundary operator. From Theorem 2.1 , one can conclude that

$$
\begin{array}{r}
E_{1}^{*, *} \cong \mathbb{R}[u] \otimes \bigotimes_{j=1}^{n}\left\{\Lambda\left(y_{j}\right) \otimes \mathbb{R}\left[\left(1, y_{j}\right)\right]\right\} \otimes \bigotimes_{i=1}^{m}\left\{\mathbb{R}\left[x_{i}\right] /\left(x_{i}^{s_{i}+1}\right) \otimes \Lambda\left(\left(1, x_{i}\right)\right)\right. \\
\left.\oplus\left(x_{i},\left(1, x_{i}\right)\right)\left[\alpha_{i}\right]^{\geq 1} /\left(\left(s_{i}+1\right) x_{i}^{s_{i}}\left(1, x_{i}\right)\right)\left[\alpha_{i}\right]\right\}
\end{array}
$$

Since $d_{1}(u)=d_{1}\left(\left(1, x_{i}\right)\right)=d_{1}\left(\left(1, y_{j}\right)\right)=0,\left(1, x_{i}\right)$ and $\left(1, y_{j}\right)$ survive to the $E_{2}$-term. The elements $u,\left(1, x_{i}\right)$ and $\left(1, y_{i}\right)$ of $E_{2}^{*, *}$ are represented by $u$, $\left(1, x_{i}\right)$ and $\left(1, y_{i}\right)$ respectively, which are in $\mathbf{N}(\Omega(X))[u]$. Since $u,\left(1, x_{i}\right)$ and $\left(1, y_{j}\right)$ are closed for the differential $D=b+u B$ of $\mathbf{N}(\Omega(X))[u]$, it follows that $d_{r}(u)=d_{r}\left(\left(1, x_{i}\right)\right)=d_{r}\left(\left(1, y_{j}\right)\right)=0$ for any $r$ (see $\left.[13]\right)$. We can define morphisms of DGAs

$$
i_{r}: \mathbb{R}[u] \otimes \mathbb{R}\left[v_{1}, \ldots, v_{n}\right] \otimes \Lambda\left(\nu_{1}, \ldots, \nu_{m}\right) \rightarrow E_{r}^{*, *}
$$

so that $i_{r}(u)=u, i_{r}\left(\nu_{i}\right)=\left(1, x_{i}\right)$ and $i_{r}\left(v_{j}\right)=\left(1, y_{j}\right)$, where $r \geq 1$. Since $d_{1}\left(x_{i}\right)=u\left(1, x_{i}\right), d_{1}\left(y_{j}\right)=-u\left(1, y_{j}\right)$ and $\operatorname{Im} d_{1} \cap E_{1}^{0, *}=0$, it follows that Ker $i_{2}=\left(v_{j} u, \nu_{i} u ; 1 \leq j \leq n, 1 \leq i \leq m\right)$. Therefore, we can conclude that the morphism of DGAs

$$
\begin{aligned}
i_{2}: A:= & \mathbb{R}[u] \otimes \mathbb{R}\left[v_{1}, \ldots, v_{n}\right] \\
& \otimes \Lambda\left(\nu_{1}, \ldots, \nu_{m}\right) /\left(v_{j} u, \nu_{i} u ; 1 \leq j \leq n, 1 \leq i \leq m\right) \rightarrow E_{2}^{*, *}
\end{aligned}
$$

is a monomorphism. Since $\operatorname{Im} d_{r} \cap E_{r}^{0, *}=0$ for any $r$, it follows that $i_{\infty}$ : $A \rightarrow E_{\infty}^{*, *}=E_{0}^{*, *}$ is a monomorphism.

Hence the algebra morphism $i: A \rightarrow H(\mathbf{N}(\Omega(X))[u], D)$ defined by $i(u)=u, i\left(v_{j}\right)=\left(1, y_{j}\right)$ and $i\left(\nu_{i}\right)=\left(1, x_{i}\right)$ is a monomorphism. By Theorem 1.1, we have Proposition 2.3.

Proof of Theorem 2.4. (1) Let $\left\{E_{r}, d_{r}\right\}$ be the cyclic spectral sequence associated with the DGA $\widetilde{\Omega}(X)$. From Theorem 2.1, we see that

$$
E_{1}^{*, *} \cong \mathbb{R}[u] \otimes \Lambda(y) \otimes \mathbb{R}[(1, y)] .
$$

Since $E_{1}^{*, *}$ is the Koszul complex with differential $d_{1}(y)=(1, y) u$, it follows that

$$
E_{2}^{*, *} \cong \mathbb{R}[u] \otimes \mathbb{R}[(1, y)] /((1, y) u) .
$$

The elements $u$ and $(1, y)$ of $\mathbf{N}(\widetilde{\Omega}(X))[u]$ representing $u$ and $(1, y)$ of $E_{2}^{*, *}$ are closed for the differential $D$. Hence we conclude that $u$ and $(1, y)$ survive to 
the $E_{\infty}$-term, that is, the spectral sequence collapses at the $E_{2}$-term ([13]). In order to determine the algebra structure of $H(\mathbf{N}(\widetilde{\Omega}(X))[u], D)$, we must solve an extension problem. Since $B(y)=(1, y)$, it follows that $(1, y) u=0$ in $H(\mathbf{N}(\widetilde{\Omega}(X))[u], D)$. Therefore we obtain the required isomorphism $\varphi_{2 l-1}$.

(2) Let $\left\{E_{r}, d_{r}\right\}$ be the cyclic spectral sequence associated with the DGA $\widetilde{\Omega}(X)$, where $q=2 l$. From Theorem 2.1, we have

$$
E_{1}^{*, *} \cong \mathbb{R}[u] \otimes\left\{\mathbb{R}[x] /\left(x^{2}\right) \otimes \Lambda((1, x)) \oplus(x,(1, x))[\alpha]^{\geq 1} /(2 x(1, x))[\alpha]\right\},
$$

where $\alpha=(1, x, x)$. Any element $z$ of $E_{1}^{*, *}$ is uniquely represented as follows:

$$
z=\sum_{i=0}^{n} \xi_{i} u^{i}, \quad \xi_{i}=\sum_{j=0}^{k_{i}}\left(\lambda_{i j} x \alpha^{j}+\mu_{i j}(1, x) \alpha^{j}\right),
$$

where $\lambda_{i j}, \mu_{i j} \in \mathbb{R}$. If $d_{1}(z)=0$, then $B\left(\xi_{i}\right)=0$ for any $i$. We obtain

$$
\sum_{j=0}^{k_{i}} \lambda_{i j}(1, x) \alpha^{j}=B\left(\xi_{i}\right)=0 .
$$

Therefore $\lambda_{i j}=0$ for any $i$ and $j$ if $z \in \operatorname{Ker} d_{1}$. Since $\operatorname{Im} d_{1}=\operatorname{Im} u B=$ $\left((1, x) \alpha^{k} u ; k \geq 0\right)$, it follows that

$$
E_{2}^{*, *} \cong \mathbb{R}[u] \otimes\left\{\Lambda((1, x)) \oplus((1, x))[\alpha]^{\geq 1}\right\} /\left((1, x) \alpha^{k} u ; k \geq 0\right)
$$

as algebras. Let $m$ be the multiplication of $\mathbf{N}(\Omega(X))[u]([9])$. By the definition, we see that $m\left(a_{1}, a_{2}\right)=S\left(a_{1}, a_{2}\right)$ if $a_{1}=\left(1, \omega_{1}, \ldots\right)$ or $a_{2}=\left(1, \gamma_{1}, \ldots\right)$. From the definition of the Connes operator $B, B\left(\left(1, \omega_{1}, \ldots\right)\right)=0$. Therefore the element $\eta_{k}$ of Proposition 4.1 is closed in $\mathbf{N}(\widetilde{\Omega}(X))[u]$, where the multiplication constructing $\eta_{k}$ is replaced by $m$. In consequence, we can take $\eta_{k} \in \mathbf{N}(\widetilde{\Omega}(X))[u]$ to represent $(1, x) \alpha^{k}$ in $E_{2}^{*, *}$. Moreover, it is possible to take the closed elements $u$ and $(1, x)$ of $\mathbf{N}(\widetilde{\Omega}(X))[u]$ as representatives of $u$ and $(1, x)$ in $E_{2}^{*, *}$ respectively. Hence the spectral sequence $\left\{E_{r}, d_{r}\right\}$ collapses at the $E_{2}$-term. From Lemma $4.2(2)$, we see that $(1, x) \cdot \eta_{k}=0$ in $\mathbf{N}(\widetilde{\Omega}(X))[u]$. It remains to solve the extension problem such that $\eta_{k} u=0$ in $H(\mathbf{N}(\widetilde{\Omega}(X))[u], D)$. Since $(1, x) \alpha^{k} u=0$ in $E_{0}^{1, *}$, the element $\eta_{k} u$ belongs to $F^{2} H^{s}(\mathbf{N}(\widetilde{\Omega}(X))[u], D)$, where $s=2 l+(4 l-2) k+1$. If $p+q$ is odd, then $E_{0}^{p, q}=0$ for any $p>0$ and $q$. This fact enables us to conclude that $F^{2} H^{s}(\mathbf{N}(\widetilde{\Omega}(X))[u], D)=0$. Hence $\eta_{k} u=0$ in $H(\mathbf{N}(\widetilde{\Omega}(X))[u], D)$. The morphism $\varphi_{2 l}$ defined by $\varphi_{2 l}(u)=u, \varphi_{2 l}((1, x))=(1, x)$ and $\varphi_{2 l}\left((1, x) \alpha^{k}\right)=\eta_{k}$ is the required isomorphism.

R e m ar k 5.1. From Theorem 2.4, we see that the morphism $i$ of Proposition 2.3 is an isomorphism if $m=0$ and $n=1$. In the case where $m=0$ and $n>1$, the element $\omega=\left(y_{j}, y_{i}\right)-\left(y_{i}, y_{j}\right)(i \neq j)$ is closed in $\mathbf{N}(\widetilde{\Omega}(X))[u]$. Since $\omega=y_{j}\left(1, y_{i}\right)-y_{i}\left(1, y_{j}\right)$, by Theorem $2.1, \omega$ is a non-zero element 
in $H(\mathbf{N}(\widetilde{\Omega}(X)), b)$. Therefore the element $\omega$ appears on the edge of the $E_{2}$-term of the cyclic spectral sequence as a non-zero element and survives to the $E_{\infty}$-term. We conclude that $\omega$ does not belong to $\operatorname{Im} i$ because the degree of $\omega$ is odd.

\section{References}

[1] W. Andrzejewski and A. Tralle, Cohomology of some graded differential algebras, Fund. Math. 145 (1994), 181-204.

[2] M. F. Atiyah and R. Bott, The moment map and equivariant cohomology, Topology 23 (1984), 1-28.

[3] E. J. Beggs, The de Rham complex on infinite dimensional manifolds, Quart. J. Math. 38 (1987), 131-154.

[4] R. Bott and L. Tu, Differential Forms in Algebraic Topology, Grad. Texts in Math. 82, Springer, New York, 1982.

[5] D. Burghelea and M. Vigué-Poirrier, Cyclic homology of commutative algebras $I$, in: Lecture Notes in Math. 1318, Springer, 1988, 51-72.

[6] H. Cartan and S. Eilenberg, Homological Algebra, Princeton University Press, Princeton, 1956.

[7] K. T. Chen, Iterated integrals of differential forms and loop space homology, Ann. of Math. 97 (1973), 217-246.

[8] E. Getzler and J. D. S. Jones, $A_{\infty}$-algebras and cyclic bar complex, Illinois J. Math. 34 (1990), 256-283.

[9] E. Getzler, J. D. S. Jones and S. Petrack, Differential form on loop spaces and the cyclic bar complex, Topology 30 (1991), 339-371.

[10] T. Goodwillie, Cyclic homology, derivations, and the free loop space, ibid. 24 (1985), 187-215.

[11] C. Hood and J. D. S. Jones, Some algebraic properties of cyclic homology groups, K-Theory 1 (1987), 361-384.

[12] J. D. S. Jones, Cyclic homology and equivariant homology, Invent. Math. 87 (1987), 403-423.

[13] D. Kraines and C. Schochet, Differentials in the Eilenberg-Moore spectral sequence, J. Pure Appl. Algebra 2 (1972), 131-148.

[14] K. Kuribayashi, On the mod p cohomology of spaces of free loops on the Grassmann and Stiefel manifolds, J. Math. Soc. Japan 43 (1991), 331-346.

[15] K. Kuribayashi and T. Yamaguchi, On additive K-theory with the LodayQuillen *-product, preprint.

[16] J. L. Loday and D. G. Quillen, Cyclic homology and the Lie algebra homology of matrices, Comment. Math. Helv. 59 (1984), 565-591.

[17] V. Mathai and D. G. Quillen, Superconnections, equivariant differential forms and the Thom class, Topology 25 (1986), 85-110.

[18] J. P. May, The cohomology of restricted Lie algebras and of Hopf algebras, J. Algebra 3 (1966), 123-146.

[19] J. McCleary, User's Guide to Spectral Sequences, Publish or Perish, Wilmington, 1985.

[20] L. Smith, Homological algebra and the Eilenberg-Moore spectral sequence, Trans. Amer. Math. Soc. 129 (1967), 58-93. 
[21] L. Smith, On the characteristic zero cohomology of the free loop space, Amer. J. Math. 103 (1981), 887-910.

[22] M. Vigué-Poirrier, Réalisation de morphismes donnés en cohomologie et site spectrale d'Eilenberg-Moore, Trans. Amer. Math. Soc. 265 (1981), 447-484.

[23] —, Sur l'algèbre de cohomologie cyclique d'un espace 1-connexe, Illinois J. Math. 32 (1988), 40-52.

[24] M. Vigué-Poirrier and D. Burghelea, A model for cyclic homology and algebraic K-theory of 1-connected topological spaces, J. Differential Geom. 22 (1985), 243-253.

Department of Applied Mathematics

Okayama University of Science

1-1 Ridai-cho

Okayama 700, Japan

E-mail: kuri@dam.ous.ac.jp

Received 21 September 1995;

in revised form 19 December 1995 